\title{
Correlation analysis of serum thyroid stimulating hormone with acute cerebrovascular disease
}

\author{
Jian Zhu', Ming Chen², Nan Li², Shaoling Yang ${ }^{2}$, Lu Xu², Yanru Wang ${ }^{2}$ and Hong $\mathrm{Li}^{2^{*}}$ (i)
}

\begin{abstract}
Background: Acute cerebrovascular disease (ACVD) could cause abnormal metabolism of thyroid hormones (TH), mostly represented as a euthyroid sick syndrome or low T3 syndrome. However, the changes in serum thyroid-stimulating hormone (TSH) are controversial. The aim of this study is to investigate the clinical significance of TSH alteration in patients with ACVD.
\end{abstract}

Method: Patients with ACVD admitted in our hospitals between January 2013 and September 2017 were enrolled in this study ( $n=245$, including 176 cerebral infarctions and 69 cerebral hemorrhages). Their thyroid hormones were measured and compared with healthy individuals $(n=75)$. The correlation of TSH with severity and prognosis of ACVD were analyzed by receiver operating characteristic curve.

Results: Serum TSH in ACVD group was higher than the control group ( $1.64 \pm 1.08 \mathrm{vs}$. $1.26 \pm 0.36 \mu \mathrm{lU} / \mathrm{mL}, P<0.05)$. The TSH levels in intermediate and severe patients with ACVD were higher than in mild patients $(1.72 \pm 1.18 \mathrm{vs}$. $2.71 \pm 0.93$ vs. $1.02 \pm 0.47 \mu \mathrm{lU} / \mathrm{mL}, P<0.05$ ). Receiver Operating Characteristic curve (ROC) of TSH in determining the severity of patients were 0.863 (Area under the curve, AUC), $1.496 \mu \mathrm{IU} / \mathrm{L}$ (optimal threshold), $76.5 \%$ (sensitivity) and 87.3\% (specificity). TSH levels in improved and unchanged groups were significantly higher than the primarily healing group ( $2.27 \pm 1.11$ vs. $2.88 \pm 1.07 \mathrm{vs}$. $0.86 \pm 0.46 \mu \mathrm{lU} / \mathrm{mL}, P<0.05)$. ROC of TSH in determining the prognosis of patients was 0.910 (AUC), $1.681 \mathrm{mlU} / \mathrm{L}$ (optimal threshold), 79.8\% (sensitivity) and 90.5\% (specificity) correspondingly.

Conclusion: Since elevated TSH in ACVD patients affects the outcome of thyroid function evaluation, it is preferable to re-check after the acute period. A correlation between a high TSH level and the severity and prognosis of ACVD was detected, but the mechanism of this correlation needs to be further studied.

Keywords: Acute cerebrovascular disease, Thyroid stimulating hormone, Cerebral infarction, Cerebral hemorrhage, Severity, Prognosis

\section{Introduction}

Cerebrovascular disease (CVD) is a common and frequently-occurring nervous system, which accounts for about $10 \%$ of all diseases, and is one of the three leading causes of human diseases. Acute cerebrovascular disease (ACVD), because of its disability rate and high mortality,

\footnotetext{
*Correspondence: HongLi_Endo@163.com

${ }^{2}$ Department of Endocrinology and Metabolism, Shanghai Tenth People's Hospital, Tongji University School of Medicine, No. 301, Middle Yan Chang Road, Shanghai 200072, China

Full list of author information is available at the end of the article
}

seriously affects the quality of life of the patients with the disease, bringing a heavy financial burden to the family and society. ACVD has many aspects of damage and influence on human body, which can not only cause the damage of nervous system function, but also can cause a series of changes in endocrine and metabolism. The pathological basis of ACVD can be caused by cerebral thrombosis, result from ischemic cerebral infarction, or can be rupture of blood vessel in brain leading to cerebral apoplexy, this kind of disease also called a stroke.

A number of studies in recent years have discovered that ACVD and many other severe non-thyroid diseases 
could cause abnormal metabolism of thyroid hormones (TH) [1-4], mostly represented as a euthyroid sick syndrome (ESS) or low T3 syndrome. However, the results of research on changes in serum thyroid-stimulating hormone (TSH) are controversial or even conflicting $[5,6]$. TSH is the most sensitive index for assessing the thyroid function and its alteration may directly affect the outcome of assessment on the thyroid function. Therefore, the aim of the present study was to define the degree of ACVD impact on TSH level by observing serum TSH changes in ACVD patients, and analyze the correlation between serum TSH change and the degree of severity of ACVD patients, in an attempt to provide experimental references for accurate assessment of the thyroid function.

\section{Materials and methods Subjects}

Written informed consent was obtained from patients under a protocol approved by the Ethics Committee of Shanghai Dachang Hospital of Baoshan District. Included in this study were 245 ACVD patients who were admitted in Dachang Hospital between January 2013 and September 2017. The diagnosis of ACVD was in accordance with the diagnosis criteria specified in the Chinese Guidelines for Prevention and Treatment of Cerebrovascular Disease [7] and confirmed by head CT scan. All patients were hospitalized for more than 14 days except deaths. The exclusion criteria were: (1) patients with thyroid and adrenal gland diseases as confirmed by medical history inquiry and B-type ultrasound; (2) patients on longterm administration of drugs such as iodine-based and anti-thyroid drugs that may affect the thyroid function; and (3) patients with severe liver and kidney dysfunction or tumors. During physical examination, additional 75 healthy individuals without a thyroid, adrenal gland, heart, liver, brain, and kidney diseases were selected from the physical examination center of the hospitals and used as control.

\section{Grouping}

Among the 245 ACVD patients, 176 patients were inflicted with acute ischemia cerebral vessels disease (AICVD), including 90 males and 86 females with a mean age of $75.03 \pm 7.25$ years, and the other 69 patients suffered from acute hemorrhagic cerebrovascular disease (AHCVD), including 39 males and 30 females with a mean age of $73.44 \pm 8.64$ years.

According to the American National Institutes of Health Stroke Scale (NIHSS), the 245 ACVD patients were graded and classified into three groups: the mild ACVD (mACVD) group (score: $\leq 6, \quad n=118$ ), the intermediate ACVD (iACVD) group (score: $7-14, n=63$ ), and the severe ACVD (sACVD) group (score: $\geq 15, n=64$ ).

The prognosis was assessed according to the scoring criteria of the clinical neurological function deficit degree of stroke patients [8] by using the following equation: (admission NIHSS score subtract NIHSS score on day 14 after admission)/admission NIHSS score * $100 \%$. Patients with a score of $91-100 \%$ fell in the basically cured group $(n=27)$; patients with a score of $46-90 \%$ fell in the significantly improved group $(n=120)$; patients with a score of $18-45 \%$ fell in the improved group $(n=59)$; patients with a score $<17 \%$ fell in the no change group $(n=24)$, and patients with an unchanged score + death fell in the deterioration group $(n=15)$. As the deterioration group included dead patients, it was unable to assess the NIHSS score on day 14 after admission, in addition, most patients in this group developed severe complications that may interfere with TSH and other THs outcomes. For these reasons, they were not included for prognosis comparison. The 75 healthy individuals in the control group included 40 males and 35 females with a mean age of $73.52 \pm 6.45$ years.

\section{Detection methods}

Serum TSH, TT3, TT4, FT3 and FT4 were measured with an automatic chemiluminescence immunoassay analyzer (ADVIA Centaur, Siemens) and appropriate reagents. The reference values of these indexes are as follows: TSH $(0.300-5.200 \mu \mathrm{IU} / \mathrm{mL})$, TT3 $(0.9-2.8 \mathrm{nmol} / \mathrm{L})$, TT4 $(65.6-179.2 \mathrm{nmol} / \mathrm{L})$, FT3 $(3.5-6.5 \mathrm{pmol} / \mathrm{L})$, and FT4 (11.9-26.0 pmol/L).

\section{Statistical analysis}

Data are expressed as mean \pm SD. TSH, TT3, TT4, FT4, and FT3 were statistically analyzed by using the SPSS20.0 software. The inter-group comparison was performed by $t$ test, and multi-group comparison was performed by analysis of variance. $P$ values $<0.05$ were considered statistically significant.

\section{Results}

General data of the included patients and healthy controls There were no significant differences in age, M/F ratio, systolic blood pressure (SBP), diastolic blood pressure (DBP), serum creatinine (CR), blood urea nitrogen (BUN), alanine aminotransferase (ALT), and aspartate aminotransferase (AST) among the cerebral infarction, cerebral hemorrhage and healthy control groups $(P>0.05)$, indicating that they were comparable (Table 1$)$. 
Table 1 Comparison of the general data between cerebral infarction, cerebral hemorrhage and healthy control groups

\begin{tabular}{lllll}
\hline Variables & Healthy control & Cerebral hemorrhage & Cerebral infarction & Normal value \\
\hline Age (years) & $73.52 \pm 6.45$ & $73.44 \pm 8.64$ & $75.03 \pm 7.25$ \\
Sex (M/F) & $40 / 35$ & $39 / 30$ & $90 / 86$ & $90.0-140.0$ \\
SBP $(\mathrm{mmHg})$ & $130.6 \pm 17.3$ & $142.3 \pm 22.5$ & $135.9 \pm 13.5$ & $60.0-90.0$ \\
DBP $(\mathrm{mmHg})$ & $88.1 \pm 15.8$ & $85.0 \pm 14.9$ & $80.8 \pm 9.6$ & $44.0-88.0$ \\
CR (Umol/L) & $61.9 \pm 6.8$ & $73.6 \pm 15.7$ & $68.3 \pm 13.7$ & $1.7-8.3$ \\
BUN (mmol/L) & $5.6 \pm 1.2$ & $5.8 \pm 1.8$ & $5.7 \pm 1.3$ & $0-60.0$ \\
ALT (U/L) & $22.8 \pm 8.9$ & $22.6 \pm 12.3$ & $24.1 \pm 10.8$ & $0-38.0$ \\
AST (U/L) & $19.9 \pm 5.2$ & $22.8 \pm 10.2$ & $21.6 \pm 6.0$ & \\
\hline
\end{tabular}

$S B P$ systolic blood pressure, $D B P$ diastolic blood pressure, $C R$ serum creatinine, $B U N$ blood urea nitrogen, $A L T$ alanine aminotransferase, $A S T$ aspartate aminotransferase

\section{Comparison of serum TH between the AVCD group and healthy control group}

Compared with the healthy control group, serum TT3, FT3, FT4 were decreased and TSH was increased in ACVD group (both $P<0.05$ ), while there was no significant difference in the level of TT4 $(P>0.05)$ (Table 2 and Fig. 1a).

\section{Comparison of TH between AICVD and AHCVD groups}

There was no significant difference in serum TT3, FT3, TT4, FT4, and TSH between AICVD and AHCVD groups (all $P>0.05$ ) (Table 3 and Fig. 1b).

\section{Comparison of TH between different severity groups} Compared with mACVD group, FT3 levels were decreased and TSH levels were increased in iACVD and sACVD groups (both $P<0.05$ ), TT3 and FT4 were decreased only in sACVD group $(P<0.05)$. Compared with iACVD group, TT3, FT3, and FT4 were decreased and TSH was increased in sACVD group (both $P<0.05$ ), whereas there was no significant difference in TT4 $(P>0.05)$ (Table 4 and Fig. 1c).

\section{TSH AUC analysis for judging the severity of ACVD}

TSH AUC for judging the severity of ACVD was 0.863 $(P<0.01)$, the optimal threshold was $1.496 \mu \mathrm{IU} / \mathrm{mL}$, sensitivity was $76.5 \%$, and specificity was $87.3 \%$, indicating that TSH level was closely correlated with the severity of ACVD (Fig. 2).

\section{Comparison of TH between groups with different prognosis}

Compared with the PC group, TSH levels in rest all groups were increased (both $P<0.05$ ), and TT3 and FT3 levels were decreased in Im and NC groups (both $P<0.05)$. Similarly, compared with SI group, TT3, and FT3 levels were decreased in Im and NC groups (both $P<0.05)$, and compared with Im group, FT3 levels were decreased in NC group (Table 5 and Fig. 1d).

TSH AUC curve analysis for judging the prognosis of ACVD The TSH ROC curve for judging the prognosis of ACVD was $0.910(P<0.01)$, the optimal threshold was 1.681 $\mu \mathrm{IU} / \mathrm{mL}$, sensitivity was $79.8 \%$, and specificity was $90.5 \%$, indicating that TSH level was to some extent correlated with the prognosis of ACVD (Fig. 3).

\section{Discussion}

TSH, a secretary hormone of adenohypophysis and a glycoprotein of $28-30 \mathrm{kDa}$, plays important roles in promoting the release and synthesis of $\mathrm{TH}$, enhancing peroxidase activity and promoting thyroglobulin synthesis and tyrosine iodization. It is accepted as the most important and sensitive index for judging the thyroid function. According to the classical theory, TSH is regulated by thyrotropin-releasing hormone (TRH), T3 and T4. However, a number of studies have demonstrated that $\mathrm{TSH}$ is regulated and interfered by a variety of factors other than the hypothalamus-hypophysis-thyroid axis, and these influencing factors may mislead the correct assessment of the thyroid function

Table 2 Comparison of serum TH between AVCD group and healthy control group

\begin{tabular}{|c|c|c|c|c|c|c|}
\hline Group & Cases $(n)$ & TT3 (nmol/L) & TT4 (nmol/L) & FT3 (pmol/L) & FT4 (pmol/L) & $\mathrm{TSH}(\mu \mathrm{IU} / \mathrm{mL})$ \\
\hline AVCD & 245 & $1.32 \pm 0.41^{*}$ & $113.00 \pm 26.89$ & $4.01 \pm 0.85^{*}$ & $16.98 \pm 3.20^{*}$ & $1.64 \pm 1.08^{*}$ \\
\hline Normal & 75 & $1.67 \pm 0.25$ & $105.86 \pm 18.11$ & $4.39 \pm 0.47$ & $18.41 \pm 3.89$ & $1.26 \pm 0.36$ \\
\hline
\end{tabular}

* $P<0.05$, compared with normal group 

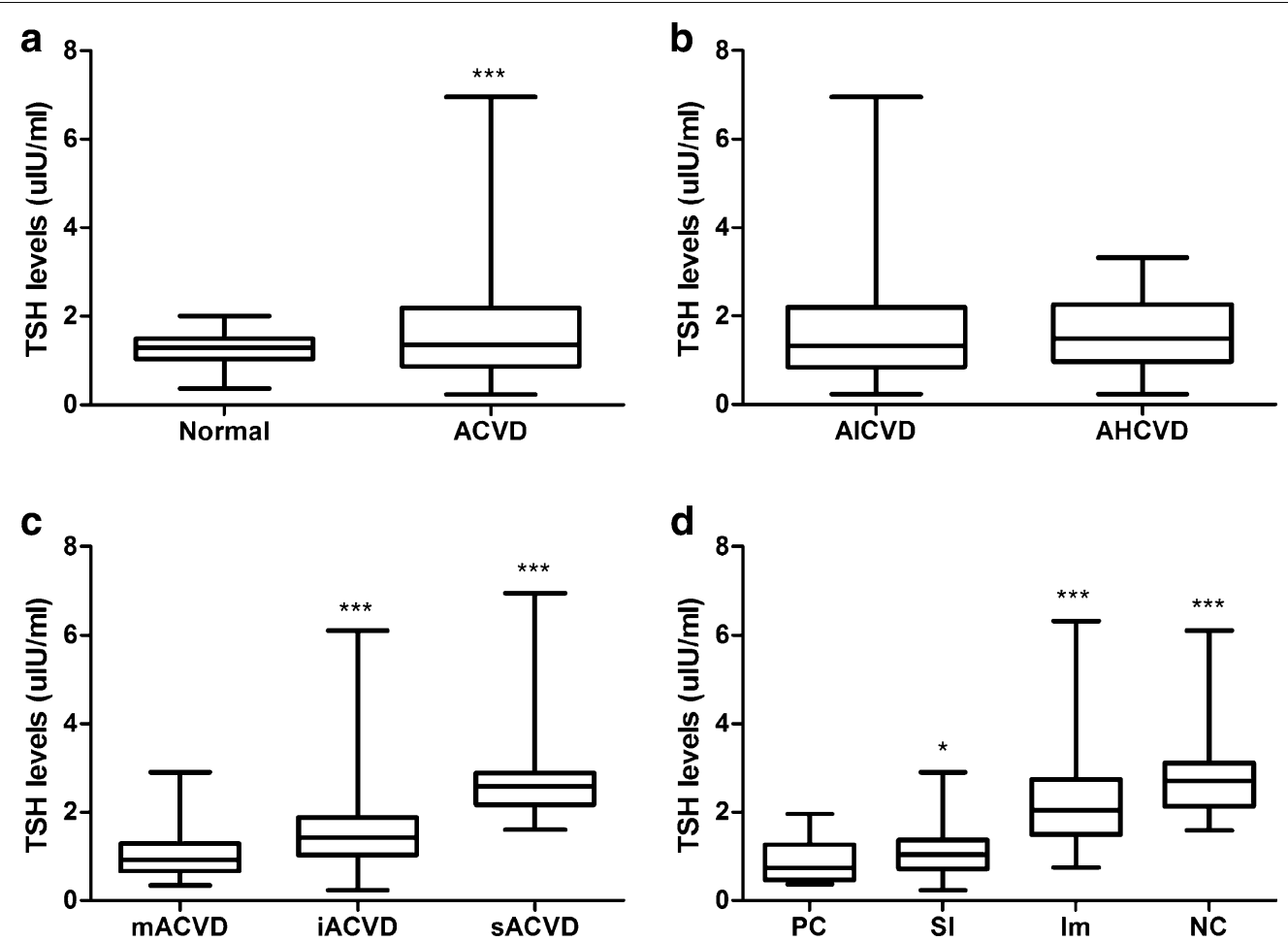

Fig. 1 The levels of TSH in different groups. a The levels of TSH were higher in the AVCD group than that in the normal group. $\mathbf{b}$ There was no significant difference in TSH levels between AIVCD and AHCVD group. c The levels of TSH were increased in iACVD and SACVD group, compared with mACVD group. $\mathbf{d}$ The levels of TSH were increased in SI, Im and NC group, compared with PC group. ${ }^{*} P<0.05$, ${ }^{* *} P<0.001$

Table 3 Comparison of serum TH between AICVD group and AHCVD group

\begin{tabular}{lllllll}
\hline Group & Cases $(\boldsymbol{n})$ & TT3 $(\mathbf{n m o l} / \mathbf{L})$ & TT4 $(\mathbf{n m o l} / \mathbf{L})$ & FT3 $(\mathbf{p m o l} / \mathbf{L})$ & FT4 $(\mathbf{p m o l} / \mathbf{L})$ & TSH $(\boldsymbol{\mu l U} / \mathbf{m L})$ \\
\hline AICVD & 176 & $1.33 \pm 0.42$ & $113.27 \pm 26.84$ & $4.08 \pm 0.87$ & $16.91 \pm 3.02$ & $1.64 \pm 1.17$ \\
AHCVD & 69 & $1.30 \pm 0.40$ & $112.32 \pm 27.21$ & $3.82 \pm 0.78$ & $17.15 \pm 3.62$ & $1.64 \pm 0.80$ \\
\hline
\end{tabular}

Table 4 Comparison of TH between different severity groups

\begin{tabular}{lclllll}
\hline Severity groups & Cases $(\boldsymbol{n})$ & TT3 $(\mathbf{n m o l} / \mathbf{L})$ & TT4 $(\mathbf{n m o l} / \mathbf{L})$ & FT3 $(\mathbf{p m o l} / \mathbf{L})$ & FT4 $(\mathbf{p m o l} / \mathbf{L})$ & TSH $(\boldsymbol{\mu l U} / \mathbf{m L})$ \\
\hline mACVD & 118 & $1.50 \pm 0.27$ & $111.91 \pm 24.75$ & $4.47 \pm 0.63$ & $16.95 \pm 2.88$ & $1.02 \pm 0.47$ \\
iACVD & 63 & $1.42 \pm 0.41$ & $120.41 \pm 25.61$ & $4.00 \pm 0.72^{*}$ & $17.99 \pm 3.60$ & $1.72 \pm 1.18^{*}$ \\
SACVD & 64 & $0.89 \pm 0.32^{* \#}$ & $107.73 \pm 30.54$ & $3.16 \pm 0.67^{* \#}$ & $16.02 \pm 3.09^{* \#}$ & $2.71 \pm 0.93^{* \#}$ \\
\hline
\end{tabular}

* $P<0.05$, compared with $\mathrm{mACVD}$ group

\# $P<0.05$, compared with iACVD group

[9]. ACVD is a neurological emergency. It can not only affect the brain function significantly but induce neuroendocrine abnormalities [10-12]. Current studies maintain that $\mathrm{TH}$ change during the acute stage of cerebrovascular disease (CVD) is mostly represented by ESS or low T3 syndrome characterized by a decrease in T3 and/or FT3. Their effect on TSH remains uncertain.
In the present study, it was found that the more severe the ACVD (the higher the NHISS score on admission), the more obvious the elevation trend of TSH. Serum TSH was higher than the reference value of $5.2 \mu \mathrm{IU} / \mathrm{mL}$ in patients with ACVD, and these patients were all in iACVD and sACVD groups, even though we found that the level of TSH in these two groups was no difference, 


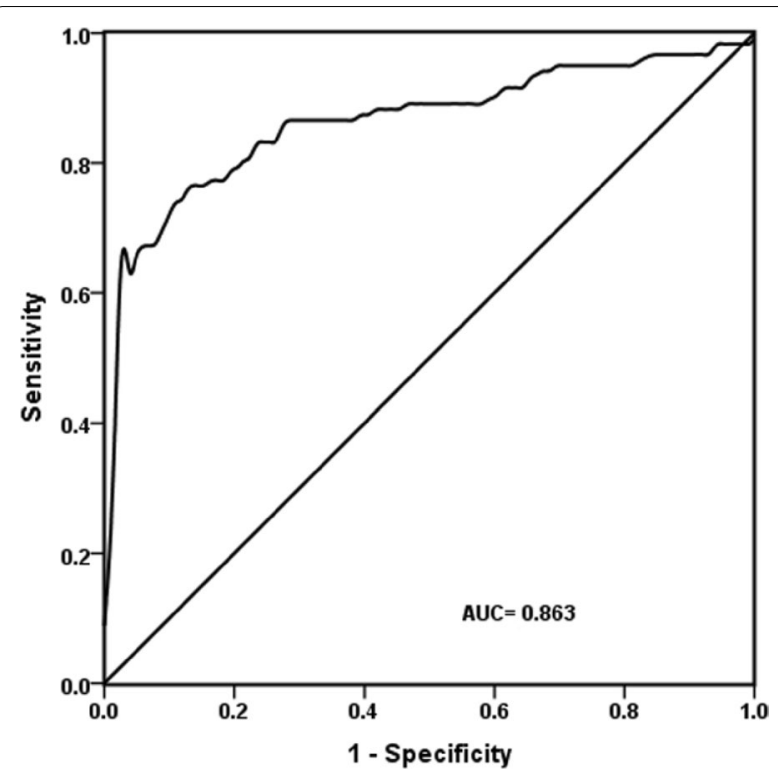

Fig. 2 ROC curve plot for the severity of ACVD judged by TSH. The area under the ROC curve (AUC) of TSH for judging the severity of ACVD was 0.863 at least indicating the amplitude of TSH change during the acute stage of CVD is correlated with the severity of the disease. In addition, patients with more pronounced elevation of TSH usually had a poorer clinical prognosis later two weeks after treatment, indicating that the severity of the condition is closely correlated with the prognosis and that the higher the TSH level on admission, the poorer the prognosis, which is consistent with the findings of other studies [13]. We postulate that ACVD is an important reason for the high serum level of TSH. The mechanism of causing ACVD-induced elevation of TSH may be explained by the following reasons. (1) Various ACVDs can induce changes in various endocrine hormones during the acute stage, such as changes in the RAS system and cortisol-ACTH axis [14]. The central regulation of thyroid function is impaired in patients with acute space occupying ischaemic stroke. These hormones may participate in TSH regulation. (2) Evidence indicated

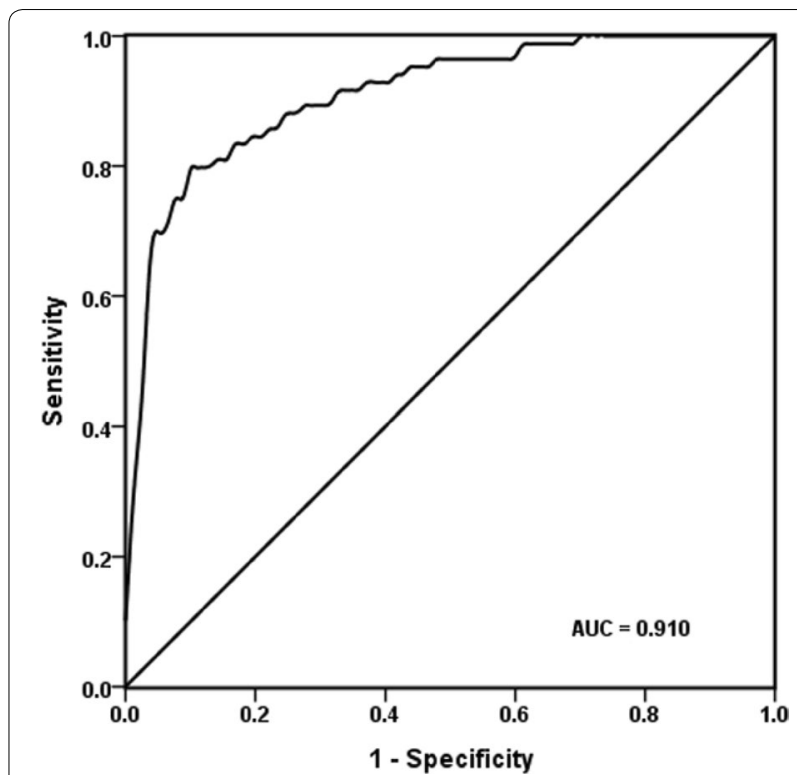

Fig. 3 ROC curve plot for the prognosis of ACVD judged by TSH. The AUC of ROC analysis for TSH was 0.910 for judging the prognosis of ACVD

that inflammatory factors, such as IL-1b, soluble IL-2 receptor, IL-6, and tumor necrosis factor- $\alpha$, have association with serum TSH levels $[15,16]$. Large amounts of inflammatory factors released during the onset of acute cardiocerebrovascular diseases may participate in TSH regulation [17]. (3) Increased intracranial pressure or cerebral circulation dysfunction due to cerebral edema during acute stroke may cause abnormal pituitary hormone secretion, leading to abnormal TSH secretion [18]. (4) The hypothalamus-pituitary-thyroid axis determines all steps of TH biosynthesis and secretion [19]. When direct lesions of hypothalamus and pituitary such as degenerative necrosis of pituitary cells occur in ACVD patients, hormones secreted or stored in pituitary cells will release into the blood, resulting in the elevation of TSH [20]. Whether these changes will produce transient or permanent injury needs to be followed up and monitored.

Table 5 Comparison of TH between groups with different prognosis

\begin{tabular}{lclllll}
\hline Group & Case $(\boldsymbol{n})$ & TT3 $(\mathbf{n m o l} / \mathbf{L})$ & TT4 $(\mathbf{n m o l} / \mathbf{L})$ & FT3 $(\mathbf{p m o l} / \mathbf{L})$ & FT4 $(\mathbf{p m o l} / \mathbf{L})$ & TSH $(\boldsymbol{\mu l U} / \mathbf{m L})$ \\
\hline PC & 27 & $1.51 \pm 0.30$ & $109.42 \pm 26.64$ & $4.51 \pm 0.59$ & $17.26 \pm 3.03$ & $0.86 \pm 0.46$ \\
SI & 120 & $1.47 \pm 0.31$ & $113.30 \pm 24.45$ & $4.34 \pm 0.70$ & $16.99 \pm 2.91$ & $1.10 \pm 0.50^{*}$ \\
Im & 59 & $1.18 \pm 0.48^{* \#}$ & $116.13 \pm 29.95$ & $3.70 \pm 0.75^{* \#}$ & $17.32 \pm 3.57$ & $2.27 \pm 1.11^{* \#}$ \\
NC & 24 & $1.01 \pm 0.32^{* \#}$ & $120.30 \pm 26.89$ & $3.20 \pm 0.57^{* \# \Delta}$ & $16.48 \pm 4.06$ & $2.88 \pm 1.07^{* \# \Delta}$ \\
\hline
\end{tabular}

* $P<0.05$, compared with $P C$ group

\# $P<0.05$, compared with $S I$ group

${ }^{\triangle} P<0.05$, compared with Im group 
In our present study, several limitations should be considered. The sample size is limited. The serum TSH levels were evaluated at a single time point. The patients included in this study were older, and the results were used to infer that the whole population of ACVD patients may be biased, which needs to be solved by further expanding the sample size and including more younger patients in the future.

\section{Conclusion}

In summary, the present study demonstrated that ACVD could induce varying degrees of TSH elevation, and therefore clinicians should be alert to the misjudgment of the impact of transient elevation of TSH on the thyroid function. Improper use of Euthyrox based on the arbitrary judgment of hypothyroidism or subclinical hypothyroidism is a hidden danger to CVD patients, especially old-aged populations. To make an accurate assessment of the thyroid function, follow-up monitoring of TSH after the acute stage of CVD is recommended.

\section{Abbreviations}

ACVD: acute cerebrovascular disease; AICVD: acute ischemia cerebral vessels disease; AHCVD: acute hemorrhagic cerebrovascular disease; AUC: area under the curve; TH: thyroid hormones; ESS: euthyroid sick syndrome; TSH: thyroid-stimulating hormone; NIHSS: National Institutes of Health Stroke Scale; mACVD: mild ACVD; iACVD: intermediate ACVD; sACVD: severe ACVD; SBP: systolic blood pressure; DBP: diastolic blood pressure; CR: serum creatinine; BUN: blood urea nitrogen; ALT: alanine aminotransferase; AST: aspartate aminotransferase; TRH: thyrotropin-releasing hormone; CVD: cerebrovascular disease; TT3: total triiodothyronine; FT3: free triiodothyronine; TT4: total thyroxine; FT4: free thyroxine; CT: computed tomography; PC: primarily cured; SI: significantly improved; Im: improved; NC: no change.
\end{abstract}

\section{Acknowledgements}

Not applicable.

\section{Authors' contributions}

JZ for study design, acquisition, analysis, interpretation of data and drafting of the manuscript; MC, NL and SY for data collection, analysis and interpretation of data; LX and YW for data collection and confirmation of manuscript. HL for study concept, design, and supervision. All authors read and approved the final manuscript.

\section{Funding}

No fund was received.

\section{Availability of data and materials}

All data generated or analyzed during this study are included in this published article.

\section{Ethics approval and consent to participate}

Written informed consent was obtained from patients under a protocol approved by the Ethics Committee of Shanghai Dachang Hospital of Baoshan District.

\section{Consent for publication}

Not applicable.

\section{Competing interests}

The authors declare that they have no competing interests.

\section{Author details}

${ }^{1}$ Department of Neurology, Dachang Hospital of Baoshan District, Shanghai 200444, China. ${ }^{2}$ Department of Endocrinology and Metabolism, Shanghai Tenth People's Hospital, Tongji University School of Medicine, No. 301, Middle Yan Chang Road, Shanghai 200072, China.

Received: 21 April 2019 Accepted: 16 October 2019

Published online: 24 October 2019

References

1. Bunevicius A, lervasi G, Bunevicius R. Neuroprotective actions of thyroid hormones and low-T3 syndrome as a biomarker in acute cerebrovascular disorders. Expert Rev Neurother. 2015;15(3):315-26.

2. Forti P, Maioli F, Coveri M, et al. Thyroid function tests and early outcomes of acute ischemic stroke in older euthyroid patients. Exp Gerontol. 2015;61:8-14.

3. Lourbopoulos A, Mourouzis I, Karapanayiotides T, Nousiopoulou E, Chatzigeorgiou S, Mavridis T, et al. Changes in thyroid hormone receptors after permanent cerebral ischemia in male rats. J Mol Neurosci. 2014;54(1):78-91.

4. Zhang Y, Meyer MA. Clinical analysis on alteration of thyroid hormones in the serum of patients with acute ischemic stroke. Stroke Res Treat. 2010;290:678-83.

5. Suda S, Muraga K, Kanamaru T, Okubo S, Abe A, Aoki J, et al. Low free triiodothyronine predicts poor functional outcome after acute ischemic stroke. J Neurol Sci. 2016;368:89-93.

6. De Groot LJ. Dangerous dogmas in medicine: the nonthyroidal illness syndrome. J Clin Endocrinol Metab. 1999;84(1):151-64.

7. Rao ML. Chinese guideline for prevention and treatment of cerebrovascular diseases. Beijing: People's Health Press; 2007. p. 18-35.

8. The 4th National Academic Conference on Cerebrovascular Diseases. Criteria for clinical neurological function deficit scoring in stroke patients. J Chin Neural. 1995;29(6):381.

9. Garber JR, Cobin RH, Gharib H, et al. Clinical practice guidelines for hypothyroidism in adults: cosponsored by the American Association of Clinical Endocrinologists and the American Thyroid Association. Endocr Pract. 2012;18(6):988-1028.

10. Wang CG. Acute cerebrovascular diseases and thyroid hormone change. J Zhejiang Clin Med Col. 2000;2(6):371-2.

11. Ambrosius W, Kazmierski R, Gupta $V$, et al. Low free triiodothyronine levels are related to poor prognosis in acute ischemic stroke. Exp Clin Endocrinol Diabetes. 2011;119(3):139-43.

12. Bustamante A, Garcia-Berrocoso T, Llombart V, et al. Neuroendocrine hormones as prognostic biomarkers in the setting of acute stroke: overcoming the major hurdles. Expert Rev Neurother. 2014;14(12):1391-403.

13. Hama S, Kitaoka T, Shigenobu M, et al. Malnutrition and nonthyroidal illness syndrome after stroke. Metabolism. 2005;54(6):699-704.

14. Schwarz S, Schwab S, Klinga K, et al. Neuroendocrine changes in patients with acute space occupying ischemic stroke. J Neurol Neurosurg Psychiatry. 2003;74(6):725-7.

15. Gomez-Zamudio JH, Mendoza-Zubieta V, Ferreira-Hermosillo A, Molina-Ayala MA, Valladares-Salgado A, Suarez-Sanchez F, de Jesus Peralta-Romero J, Cruz M. High thyroid-stimulating hormone levels increase proinflammatory and cardiovascular markers in patients with extreme obesity. Arch Med Res. 2016;47(6):476-82.

16. Milani AT, Khadem-Ansari MH, Rasmi Y. Effects of thyroid-stimulating hormone on adhesion molecules and pro-inflammatory cytokines secretion in human umbilical vein endothelial cells. Res Pharm Sci. 2018;13(6):546-56.

17. Adler SM, Wartofsky L. The nonthyroidal illness syndrome. Endocrinol Metab Clin North Am. 2007;36(3):657-72.

18. Suzuki Y, Nanno M, Gemma R, et al. Plasma free fatty acids, inhibitor of extrathyroidal conversion of T4 to T3 and thyroid hormone binding inhibitor inpatients with various nonthyroidal illness. Endocrinol Jpn. 1992:39(5):445-53. 
19. Ortiga-Carvalho TM, Chiamolera MI, Pazos-Moura CC, Wondisford FE. Hypothalamus-pituitary-thyroid axis. Compr Physiol. 2016;6(3):1387-428.

20. Seo $C$, Kim S, Lee M, et al. Thyroid hormone replacement reduces the risk of cardiovascular diseases in diabetic nephropathy patients with subclinical hypothyroidism. Endocr Pract. 2018;24(3):265-72.

\section{Publisher's Note}

Springer Nature remains neutral with regard to jurisdictional claims in published maps and institutional affiliations.
Ready to submit your research? Choose BMC and benefit from:

- fast, convenient online submission

- thorough peer review by experienced researchers in your field

- rapid publication on acceptance

- support for research data, including large and complex data types

- gold Open Access which fosters wider collaboration and increased citations

- maximum visibility for your research: over 100M website views per year

At BMC, research is always in progress.

Learn more biomedcentral.com/submissions 